\title{
AVALIAÇÃO DA MACROPOROSIDADE E DA DENSIDADE DE PARTÍCULAS DO SOLO ATRAVÉS DA MICROTOMOGRAFIA DE RAIOS-X(1)
}

\author{
A. MACEDO(2) \& S. CRESTANA ${ }^{(2)}$
}

\begin{abstract}
RESUMO
A Ciência do Solo procura compreender e modelar os fenômenos ocorridos no solo, princi palmente na zona não saturada. Como o entendimento de muitos desses fenômenos exige determi nações em escalas que permitam incorporar aos modelos a variabilidade de poros e agregados, desenvolveu-se, na Embrapa Instrumentação Agropecuária, um tomógrafo de raios- $X$, de resolução micrométrica, com o objetivo de se obterem imagens, de forma não-destrutiva, de amostras de solo, com resolução espacial igual ou inferior a $100 \mu \mathrm{m}$. Foi possível projetar e construir um equipamento, de alta resolução, com baixo custo, comparativamente aos equipamentos comerciais disponíveis, cujos preços são proi bitivos para aplicações em solo. Obtiveram-se imagens de amostras de solos com estrutura deformada, compostos por agregados de 212 a $250 \mu \mathrm{m}$, nos quais foram identificados poros medindo $100 \mu \mathrm{m}$ ou menos. Poros dessas dimensões foram visualizados, também, em tomografias de amostras compostas de grãos de arei a de $1 \mathrm{~mm}$ e capi lares de vidro com diâmetros internos de 100, 200 e $300 \mu \mathrm{m}$. Tomografias de amostras não deformadas de solos evidenciaram, além de poros de 200 a $800 \mu \mathrm{m}$, partículas de alta densidade não-detectáveis com a tomografia de resolução milimétrica.
\end{abstract}

Termos de indexação: tomografia, microtomografia, solo, raio-X, agricultura, meios porosos.

(1) Parte da Tese de Doutorado do primeiro autor. Projeto financiado pela E mbrapa/I nstrumentação Agropecuária (Projeto 12.0.94.093) e pela FAPESP (Processo 90/3773-7). Escola de Engenharia de São Carlos - Centro de Recursos Hídricos e Ecologia Aplicada USP. Recebido para publicação em maio de 1998 e aprovado em maio de 1999.

(2) Pesquisador da Embrapa Instrumentação Agropecuária. Rua XV de Novembro, 1452, Caixa Postal 741. CEP $13560-970$ São Carlos (SP). E-mail: alvaro@cnpdia.embrapa.br. 


\title{
SUMMARY: MACROPOROSITY AND SOIL PARTICLE DENSITY EVALUATION USING X-RAY MICROTOMOGRAPHY
}

\begin{abstract}
Soil Sciencedeals with theunderstanding and modelling of thephenomena occurring in soils, mainly thoserelated to the non-saturated zone. Since understanding most of those phenomena requires determination of parameters in scales all owing the incorporation of pore and aggregate variability to the models, a microscopic scale X-ray tomograph was built at E mbrapa Agricul ture Instrumentation. The aim was to generate non-destructive images of soil samples, within at least $100 \mu \mathrm{m}$ spatial resolution. It was possi bl e to design and construct a low cost equi pment as compared to commercial ly avail ablescanners, whose prices are prohi bitiveto soil studies. Tomographs of disturbed soi ls showing 212 to $250 \mu \mathrm{m}$ aggregates wereobtained. Pores with $100 \mu \mathrm{m}$ sizeor less wereobserved. Pores with thesame sizecoul d bedetected in tomographs of samples containing $1 \mathrm{~mm}$ sand grains and capillaries with internal diameter of 100, 200 and $300 \mu \mathrm{m}$. Tomographs of undisturbed soil samples areal so shown with pores ranging from 200 to $800 \mu \mathrm{m}$ and high density partides.
\end{abstract}

Index terms: tomography, mi crotomography, soil, X-ray, agriculture, porous media.

\section{INTRODUÇÃO}

Os solos e os recursos hídricos têm sido submetidos, cada vez mais, às agressões da pol uição de fontes dispersas e às conseqüências de manejos que, em vários casos, não levam em consideração a sustentabilidade do sistema. Para classificar e quantificar os efeitos dos agentes de alteração, necessita-se da utilização de novas ferramentas e métodos modernos de investigação, que permitam, principalmente, manejá-los adequadamente, de forma a serem evitados (Crestana, 1994).

Como as técnicas convencionais têm-se mostrado limitadas diante da complexidade dos mecanismos físicos, químicos e bi ológi cos que atuam nosol o, novos métodos, originários de diversas áreas do conhecimento, têm sido utilizados pela primeira vez em solos (Crestana et al., 1996), a saber: as espectroscopias de ressonância magnética nuclear e paramagnética eletrônica; as espectroscopias de infravermelho, ultravioleta visível e PIXE; a espectroscopia de fluorescência; a espectroscopia de plasma; a tomografia sísmica; o sensoriamento remoto; a reflectometria no domínio do tempo; a voltametria e as microscopias de varredura, de tunelamento e de força atômica. Crestana \& Vaz (1997) apresentam um panorama do emprego da instrumentação na pesquisa em manejo e conservação do solo.

Uma técnica que tem obtido boa repercussão, considerando a resolução e a possibilidade de investigar o interior de objetos opacos sem necessidade de seccioná-los (deforma não invasiva), é a Tomografia Computadorizada (TC). A TC pode ser de raios- $X$, raios $\gamma$ e Ressonância Magnética Nuclear (RMN), entreoutras formas. A TC por RMN, entretanto, mostra fortes restrições para seu uso em mei os porosos que contêm materiais paramagnéticos (Crestana \& Nielsen, 1990).

E mbora a TC de raios-X e de radiação $\gamma$ tenhamse mostrado muito valiosas como técnicas recentes do uso de imagem para estudos não-invasivos em solos, os resultados, atéo momento, têm sido obtidos numa escala da ordem de milímetros (Petrovic et al., 1982; Hainsworth \& Aylmore, 1983; Cruvinel et al., 1990; Crestana et al., 1996). Muitas respostas, porém, devem ser obtidas, preferencialmente, em nível micrométrico. Alguns resultados expressivos foram al cançados, na escala submilimétrica. Este é o caso, por exemplo, de uma microtomografia com resolução espacial igual a $20 \mu \mathrm{m}$, obtida por Crestana et al. (1996). No entanto, este equipamento está restrito a aplicações militares e industriais e com elevado custo, sendo aplicado à análise de objetos com densidade el evada e de grandes dimensões, se comparados com amostras de solo. Outros resultados foram apresentados por Flannery et al. (1987), Cesareo et al. (1993, 1994), os quais estiveram restritos a amostras com secções iguais ou inferiores a $3 \times 3 \mathrm{~mm}^{2}$, impedindo sua aplicação em amostras desoloindeformadas. Por esse motivo, construiu-se, na Embrapa Instrumentação Agropecuária, um tomógrafo com capacidade de obter imagens de amostras de solo indeformadas, com resolução micrométrica, a um custo baixo, quando comparado com os tomógrafos comerciais, cujo custo se situa na ordem de meio milhão a um milhão de dólares, enquanto o equipamento aqui apresentado teve um custo em torno de US\$ 70 mil.

A microtomografia traz vantagens sobre técnicas invasivas, por possi bilitar o exame das amostras sem alterar as suas características. Entende-se por 
técnicas invasivas aquelas que podem trazer alteração na estrutura da amostra, tais como: dimensões dos agregados, dimensões e distribuição dos poros e distribuição do conteúdo de água e solutos. A porosimetria por intrusão de mercúrio, por exemplo, é uma técnica amplamente utilizada, trazendo informações importantes sobre a distribuição de poros do solo. Todavia, enfrenta problemas práticos graças à sua invasibilidade (Kutilek \& Nielsen, 1994).

Breve explanação sobre o método tomográfico pode ser encontrada em Crestana et al . (1992) e Vaz et al. (1992). O detal hamento matemático do al goritmo de reconstrução pode ser visto em Rosenfeld \& Kak (1982), entre outros.

O objetivo deste trabal ho foi o desenvol vimento de um tomógrafo de raios- $X$, com resolução micrométrica, e sua aplicação para investigação de macroporos e determinação da densidade de partículas do solo.

\section{MATERIAL E MÉTODOS}

O experimento foi desenvolvido nos laboratórios da Embrapa Instrumentação Agropecuária, utilizando o tomógrafo de resolução micrométrica, desenvolvido neste Centro, constituído de: sistema de raios-X da Philips composto de gerador de $3 \mathrm{~kW}$, tensão máxima de saída 160 kV, painel de controle el etrônico, painel decomando etubo de 3 kW, comalvo de tungstênio; cadeia nuclear composta de detector de iodeto de sódio ativado com tálio - $\mathrm{Nal}$ ( $\mathrm{TI}$ ) -, fonte de al ta tensão de 50 a $3.000 \mathrm{~V}$, fonte de baixa tensão com potência de $160 \mathrm{~W}$ e placa multicanal; mesa composta de estágio linear, com resolução de 1,25 $\mu \mathrm{m}$, estágio rotativo, com resolução de $0,01^{\circ}$ e um controlador para cada estágio; colimadores de chumbo ou de resina epóxi com $\mathrm{PbO}_{2}$, nos diâmetros de 215, 100 e $60 \mu \mathrm{m}$. O diagrama de blocos deste tomógrafo pode ser visto na figura 1.

Nas tomografias, foram utilizadas as seguintes amostras: uma amostra de Podzólico Vermel hoAmarelo, da F azenda Galvão, Santa Bárbara (SP), com $19 \%$ de argila, $18 \%$ de silte e $63 \%$ de areia; uma amostra de Latossolo Vermel ho-E scuro, da Fazenda Canchim, da Embrapa Pecuária doSudeste, em São Carlos (SP), com 39\% de argila, $8 \%$ de siltee $53 \%$ de areia; uma amostra de sol onão classificado, de uma área na estrada I tirapina - Dois Córregos, no estado de São Paulo, com $66 \%$ de argila, $7 \%$ de silte e $27 \%$ de areia; uma amostra com grãos de areia de 1 a $2 \mathrm{~mm}$ e capilares de vidro com diâmetros de $0,1,0,2$ e 0,3 mm e uma amostra com grãos de areia de 0,5 a $1,0 \mathrm{~mm}$.

$\mathrm{Na}$ calibração das unidades tomográficas em coeficientes de atenuação linear, empregou-se o método desenvolvido por Crestana et al . (1992). Essa calibração foi feita na energia de $58,5 \mathrm{keV}$, sendo utilizadas as seguintes amostras homogêneas: acrílico, água, ál cool etílico, Latossol o Roxo distrófico (passado em peneira $53 \mu \mathrm{m}$ para homogeneizá-la), nylon, Podzólico Vermelho (peneirado como a amostra anterior), PVC, teflon e microesferas de vidro (granulometria de 105 a $149 \mu \mathrm{m}$ ). Foram col etados dados de número de fótons atravessando nove pontos diferentes de cada amostra, para o cálculo de $\mu$. Obteve-se a correlação apresentada na equação 1 (Macedo et al., 1999).

$$
\mu=0,9697 \text { UT +0,0036, com } R^{2}=0,9992
$$

$\mathrm{Na}$ calibração do tomógrafo, em termos de unidades tomográficas versus densidade dos minerais queo compõem, utilizaram-seamostras dos seguintes minerais, por estarem entre os

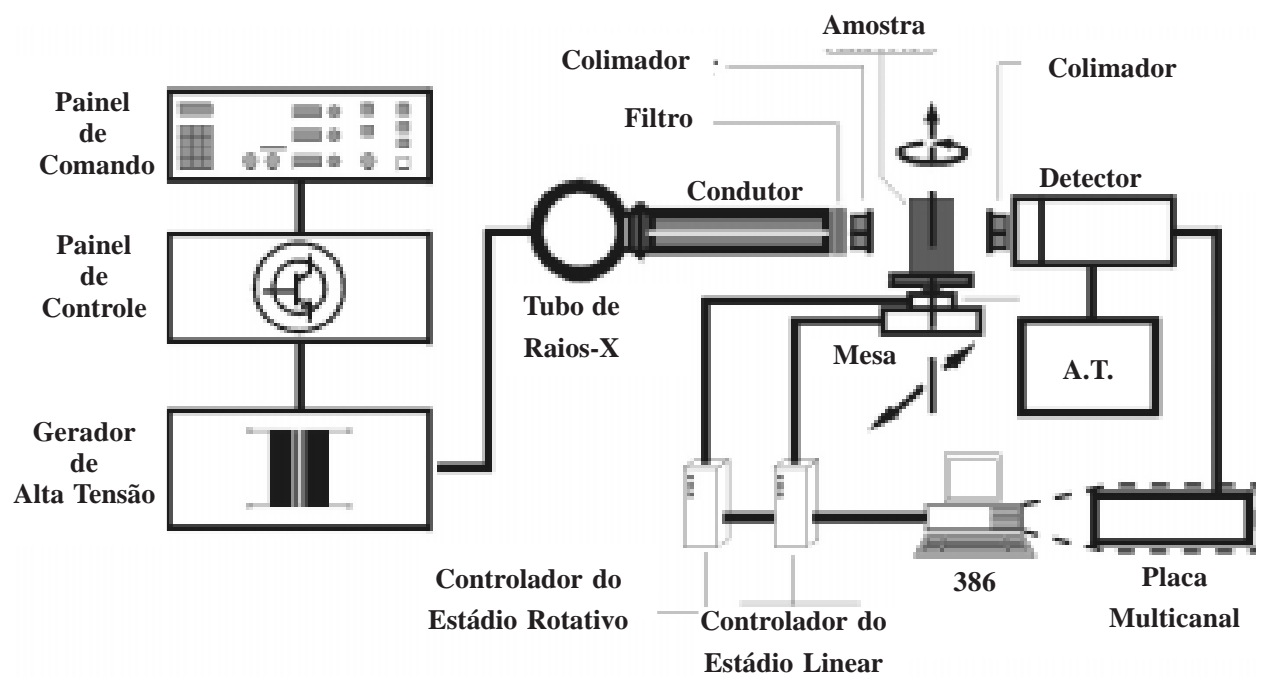

Figura 1. Diagrama de blocos do tomógrafo com resolução micrométrica. 
componentes mais freqüentes dos solos do estado de São Paulo: hematita, caulinita, quartzo, calcita, ortoclásio eal bita. Para obter amostras homogêneas e com geometria conhecida, neste procedimento de calibração, as amostras de minerais foram moídas em almofariz, passadas em peneiras com malha de $105 \mu \mathrm{m}$ e colocadas em recipiente cilíndrico de acrílico com diâmetro interno de 10,81 mm, altura de 15,20 mm e parede com espessura de 0,92 $\mathrm{mm}$.

A densidadeéuma quantificação importante que se pode extrair da imagem tomográfica. $\mathrm{Na}$ tomografia milimétrica, obtêm-se, a partir das imagens, os valores das unidades tomográficas de amostras de solo com densidades conhecidas. Produz-se, assim, um gráfico da densidadeem função das unidades tomográficas, em que valores médios são devidos à resolução empregada (Crestana et al., 1985, 1992). Na tomografia micrométrica, entretanto, surgem detal hes característicos dos minerais constituintes do solo. São necessárias, então, duas calibrações: uma para valores globais, seguindo o mesmo procedimento desenvol vido por Crestana et al. (1985, 1992), e outra para partículas ou cristais dos minerais. Para efetuar tal calibração, são escol hidos al guns minerais mais comuns ao sol o que se pretenda estudar. Podem ser utilizadas, também, al gumas misturas desses minerais, para obter maior número de pontos na curva. U ma alternativa para esse procedimento seria analisar o solo, determinando-se seus minerais e, a partir dessa informação, fazer as escolhas. Todavia, desde que, com o método proposto, sejam obtidos pontos suficientes para tal calibração, essa forma é mais rápida e suficiente.

Para determinar o coeficiente deatenuaçãolinear dos minerais $\left(\mu_{\text {cristal }}\right)$, mede-se a atenuação da radiação $X$ pelas amostras, que se apresentam na forma de pó, e calcula-se o seu coeficiente de atenuação em massa $\left(\mu_{\mathrm{m}}\right)$, conforme indicado na equação 2 . Obtém-se o $\mu_{\text {cristal }}$ por meio da equação 4.

$$
\mu_{\mathrm{m}}=\frac{\mu}{\rho_{\mathrm{g}}}
$$

em que $\rho_{g}$ é a densidade do solo da amostra e $\mu$ é o coeficiente de atenuaçãolinear da amostra, na forma de pó, dado por:

$$
\mu=\frac{1}{\mathrm{x}} \cdot \ln \left(\frac{\mathrm{I}_{\mathrm{Oa}}}{\mathrm{I}}\right)
$$

sendo $x$ a espessura da amostra, $I_{\text {oa }}$ a intensidade do feixe de fótons que atravessa o recipiente vazio e I a intensidade do feixe de fótons que atravessa o recipiente com a amostra.

$$
\mu_{\text {cristal }}=\mu_{\mathrm{m}} \rho_{\mathrm{s}}
$$

em que $\rho_{\mathrm{s}}$ é a densidade das partículas da amostra.

Na medição do coeficiente de atenuação linear, utilizam-se, geralmente, amostras com faces planas paralelas dispostas perpendicularmente ao feixe. No caso do tomógrafo de resolução mi crométrica, tornase difícil conhecer, de forma precisa, a direção do feixe, o que compromete a sua perpendicularidade em relação às faces planas e, portanto, a determinação de $x$ é inexata. Para contornar essa limitação, foram usados um recipiente cilíndrico e amostras em pó. Não se perde informação com esse procedimento, dada a relação linear entre os coeficientes $\mu$ e $\mu_{m}$. A amostra em pó é col ocada no recipiente e faz-se uma varredura com passos de $10 \mu \mathrm{m}$. A menor contagem defótons, se não houvesse a flutuação devida à estatística de Poisson, deveria ocorrer exatamente no centro da amostra e na espessura $x$, que corresponderia ao diâmetro interno do recipiente.

Para minimizar o erro causado por tal flutuação, tomou-se a média de três pontos, ou seja, o menor número de fótons e os valores das posições adjacentes. Em outras palavras, I é a média aritmética de $\mathrm{I}_{\mathrm{c}-1}, \mathrm{I}_{\mathrm{C}} \mathrm{el}_{\mathrm{c}+1}$. (Figura 2). Comoa amostra pode não estar homogeneamente distribuída, em termos de densidade, tanto vertical como horizontalmente, as medidas foram feitas em quatro planos horizontais distando $1 \mathrm{~mm}$ entre si e em três direções em cada plano $(\theta=0 \circ, \theta=60$ ㅇ e $\theta=120$ ). Considerou-se como feixe livre a média das três primeiras contagens de fótons fora da amostra e $\mathrm{I}_{\mathrm{ao}}=\mathrm{I}_{\mathrm{o}}$. $\exp \left(-\mu_{\mathrm{ac}} \mathrm{X}_{\mathrm{ac}}\right)$, em que $\mu_{\mathrm{ac}}=0,225 \mathrm{~cm}^{-1}$ é o coeficiente de atenuação linear para o acrílico, em $58,5 \mathrm{keV}$, e $\mathrm{x}_{\mathrm{ac}}=0,185 \mathrm{~cm}$ é a espessura de acrílico que o feixe de fótons atravessa.

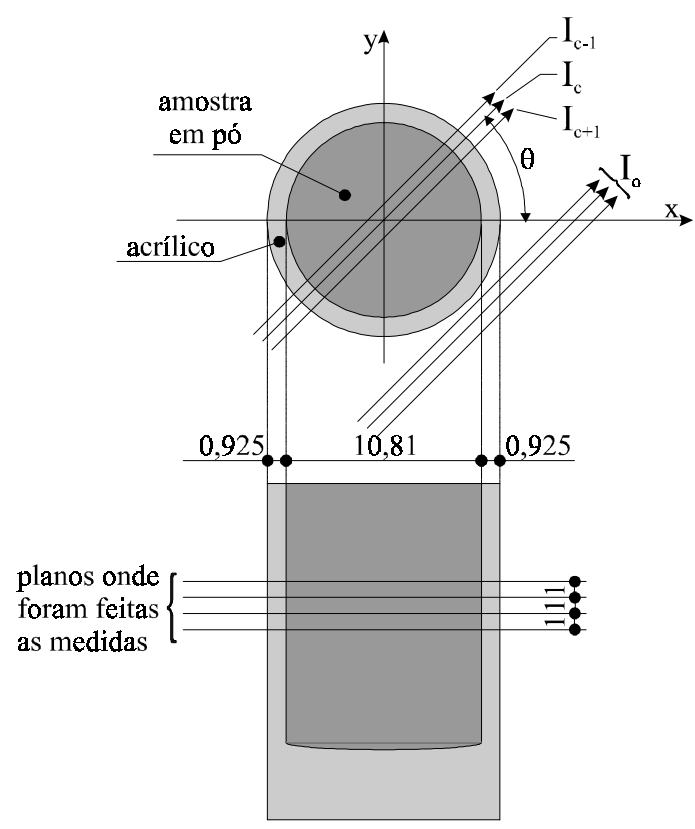

Figura 2. Diagrama mostrando as posições em que foram feitas as medidas para o cálculo dos coeficientes de atenuação linear, para calibração das tomografias em densidade das partículas. 
Quanto à preparação das amostras de solos, trabalhou-se com amostras deformadas e não deformadas. No primeiro caso, as amostras foram peneiradas e col ocadas em recipientes cilíndricos de acrílico, com diâmetros internos de 4,8 e 10,81 mm. Os agregados de Podzólico Vermel ho-Amarel oforam separados em peneiras com malhas de 0,212 e $0,250 \mathrm{~mm}$. Para a areia, utilizaram-se peneiras com mal ha de 1,0 a 2,0 mm e com mal ha de 0,5 a 1,0 mm.

No caso de amostras não deformadas, foram utilizados pequenos torrões de solo, obtidos por fragmentação manual ou por seccionamento com lâmina. Não se fez nenhum tipo de impregnação, para evitar qualquer deformação interior por arraste deagregados ou alteraçãona dimensão e distri buição dos poros.

\section{RESULTADOS E DISCUSSÃO}

A calibração do tomógrafo, correlacionando a densidade das partículas com o coeficiente de atenuaçãolinear, ésumariada no quadro 1, onde são apresentados estes dados para as amostras utilizadas na calibração(3) . A correlação entre $\rho_{\mathrm{s}}$ e $\mu$ pode ser vista na equação 5 . Para as misturas, as densidades reais foram extrapoladas, cal culando-se a média ponderada dos valores dos minerais utilizados na sua composição. A não-utilização desse artifício resultaria em $\rho_{\mathrm{s}}=0,6203 \mu+2,0905$, com $\mathrm{R}^{2}=0,9922$. Porém, a diferença entre as duas equações éinferior a 1\%, além de seobterem apenas pontos próximos aos extremos do intervalo 0,654 a $5,341 \mathrm{~cm}^{-1}$.

$$
\rho_{\mathrm{s}}=0,6139 \mu+2,1172, \text { com } \mathrm{R}^{2}=0,9898
$$

Os resultados seguintes mostram as imagens de amostras nas condições já descritas, sendo visíveis os poros. Na figura 3, vêse a tomografia de uma amostra de Podzólico Vermelho, peneirado, com agregados de 212 a $250 \mu \mathrm{m}$. Os poros são visíveis e suas dimensões no plano em análise foram medidas no programa de reconstrução e visualização, apresentando valores da ordem de $100 \mu \mathrm{m}$. Utilizaram-se energia de 58,5 keV, colimador de $60 \mu \mathrm{m}$, tempo de contagem de 10s e feixe livre de 42.000 fótons. O tempo de contagem é o tempo durante o qual são contabilizados os fótons para cada posição da translação. O feixelivre citado representa o número de fótons contabilizado durante o tempo de contagem sem a presença da amostra.

$\mathrm{Na}$ figura 4, pode ser vista a microtomografia de uma amostra constituída de grãos de areia de, aproximadamente, um milímetroe capilares devidro

(3) Amostras cedidas pelo Prof. J osé E duardo Rodrigues, do Departamento de Geotecnia da Escola de Engenharia de São Carlos/USP.
Quadro 1. Densidade dos sólidos $\left(\rho_{s}\right)$ e coeficiente de atenuação linear $(\mu)$ de amostras de minerais, para a energia de $58,5 \mathrm{keV}$

\begin{tabular}{|c|c|c|}
\hline Amostra & $\mu$ & $\rho_{s}^{(1)}$ \\
\hline & $\mathrm{cm}^{-1}$ & $\mathrm{~g} \mathrm{~cm}^{-3}$ \\
\hline Hematita & 5,090 & 5,27 \\
\hline Caulinita & 0,756 & 2,62 \\
\hline Quartzo & 0,788 & 2,65 \\
\hline Mistura $1^{(2)}$ & 1,921 & 3,47 \\
\hline Mistura 2 & 2,971 & 3,92 \\
\hline Mistura 3 & 3,968 & 4,50 \\
\hline Calcita & 1,283 & 2,71 \\
\hline Ortoclásio & 0,836 & 2,57 \\
\hline Albita & 0,706 & 2,59 \\
\hline
\end{tabular}

(1) Calculada pelo método do picnômetro (EMBRAPA, 1997). (2) $31,9 \%$ de hematita e $68,1 \%$ de caulinita. (3) $50,2 \%$ de hematita e $49,8 \%$ de caulinita. ${ }^{(4)} 71,1 \%$ de hematita e $28,9, \%$ de caulinita.

com diâmetros internos de 100, 200 e $300 \mu \mathrm{m}$. Notam-se canais entre grãos com dimensões iguais ou inferiores a $100 \mu \mathrm{m}$. A energia utilizada nesta tomografia foi de 58,5 keV, o colimador de $60 \mu \mathrm{m}$, o tempo de contagem de 10s e o feixe livre de 39.700 fótons.

A amostra da figura 5 é constituída de grãos de areia situados na faixa de 0,5 a $1 \mathrm{~mm}$, col ocados num cilindro com 10,81 mm de diâmetro interno. Os contornos dos grãos podem ser vistos, bem como os poros na dimensão do pixel, de $59 \mu \mathrm{m}$. A energia do feixefoi de $61 \mathrm{keV}$, o colimador de $60 \mu \mathrm{m}$, otempo de contagem de $6 s$ e o feixe livre de 27.000 fótons.

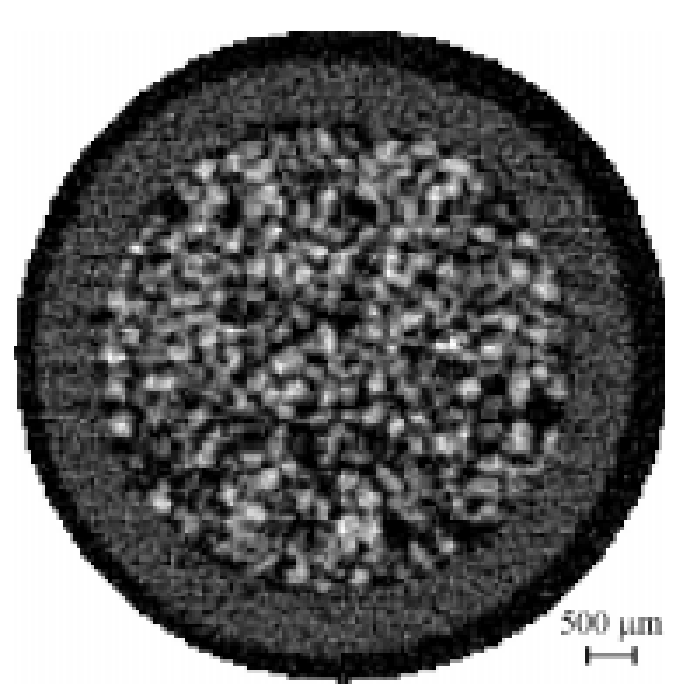

Figura 3. Tomografia de amostra deformada de Podzólico Vermelho, apresentando agregados de 212 a $250 \mu \mathrm{m}$. As regiões mais escuras representam o ar. 


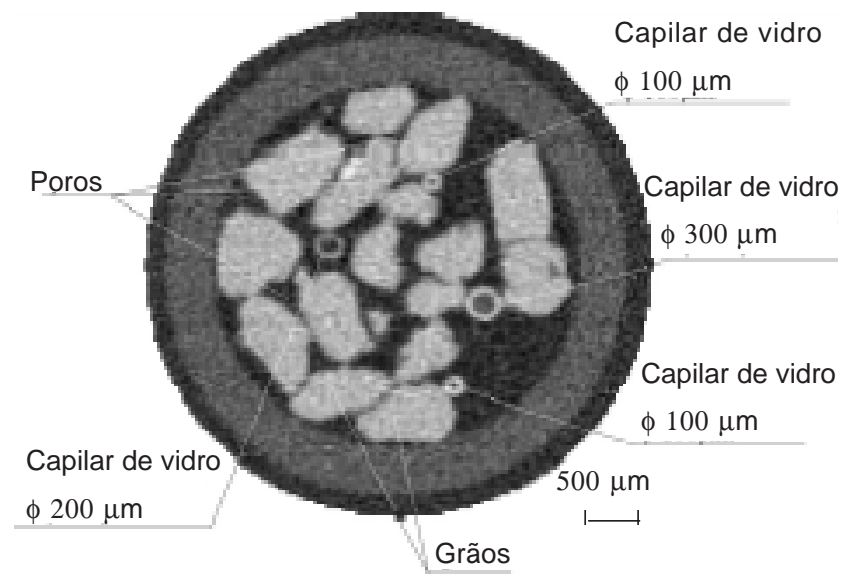

Figura 4. Tomografia de uma amostra de grãos de areia de aproximadamente $1 \mathrm{~mm}$, capilares de vidro, sendo dois de 100, um de 200 e um de $300 \mu \mathrm{m}$.

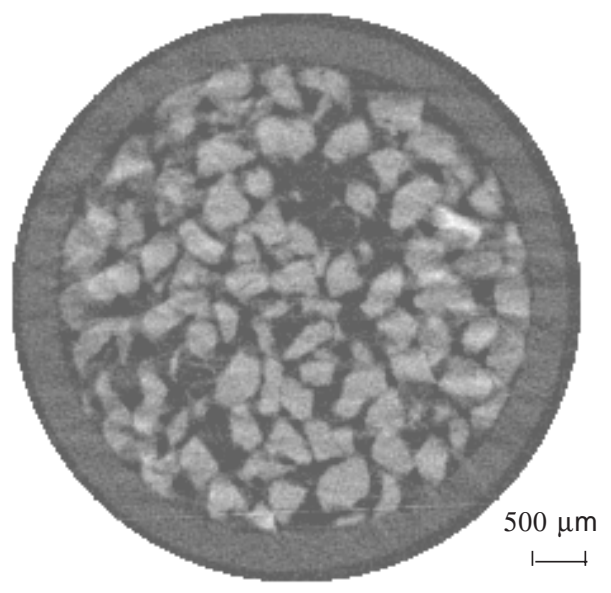

Figura 5. Tomografia de uma amostra de grãos de areia de 0,5 a $1 \mathrm{~mm}$ em recipiente com 10,81 mm de diâmetro interno.

Nos resultados com torrões, podem ser vistas as tomografias mostradas nas figuras 6 e 7 . $\mathrm{Na}$ primeira, tem-seum pequeno torrão de um $L$ atossolo Vermelho-Escuro, colocado no interior de um recipiente de acrílico com 4,8 mm de diâmetro interno. Podem ser notados, nessa imagem, dois macroporos com dimensões da ordem de $300 \mathrm{~mm}$ e alguns pontos com densidade mais el evada. A unidade tomográfica máxima encontrada foi de 1,465 , que corresponde, pela apli cação das equações 1 e 5, a $\mu=1,424 \mathrm{~cm}^{-1}$ ea $\rho_{\mathrm{s}}=2,991 \mathrm{~g} \mathrm{~cm}^{-3}$. O processo de aquisição er econstrução resol veu, espacialmente, de forma satisfatória, essa imagem, podendo-se visualizar com nitidez os contornos do torrão, a região com ar no espaço não ocupado pela amostra e os contornos e espaços vazios formados entre este e o recipiente. Foram utilizados energia de $58,5 \mathrm{keV}$, colimador de $100 \mu \mathrm{m}$, tempo de contagem de $5 \mathrm{~s}$ e feixe livre de 38.000 fótons. A figura 7 mostra a microtomografia deum fragmento de torrão desol o(4) . Poros de, aproximadamente, 200 a $800 \mu \mathrm{m}$ podem ser vistos, bem como as interconexões entre al guns deles. A presença de pontos com alta densidade também pode ser notada. A unidade tomográfica máxima encontrada foi de 3,167 , correspondendo a $\mu=3.075 \mathrm{~cm}^{-1}$ ea $\rho_{\mathrm{s}}=4,005 \mathrm{~g} \mathrm{~cm}^{-3}$.

Na figura 8, observou-se a tomografia de uma amostra construída com al guns cristais para sefazer uma comparação entre os valores obtidos pelo método aqui proposto e as densidades conhecidas dos materiais. Foram utilizados, conformeindicado, cristais de quartzo, ortoclásio, hematita e calcita. O quadro 2 mostra os val ores obtidos, com erro máximo de $4,7 \%$, ocorrido para o quartzo.

A microtomografia traz um novo enfoque e uma nova alternativa à investigação das características físicas do solo. Os conceitos densidade e umidade, por exemplo, consagrados para a macroescala, não podem ser estendidos para a microescala. Isto, pelo menos, não é possível ou evidente até o momento, a menos que se consiga fazer uma correlação entre escalas. Na macroescala, enquanto se avaliam massa e volume, na microescala utilizam-se conceitos de espaço poroso, vazios, densidades de poros, distribuição de poros, espaços ocupados por água, solutos, gases e assim por diante. Necessita-se, portanto, equacionar como conectar esses dois níveis

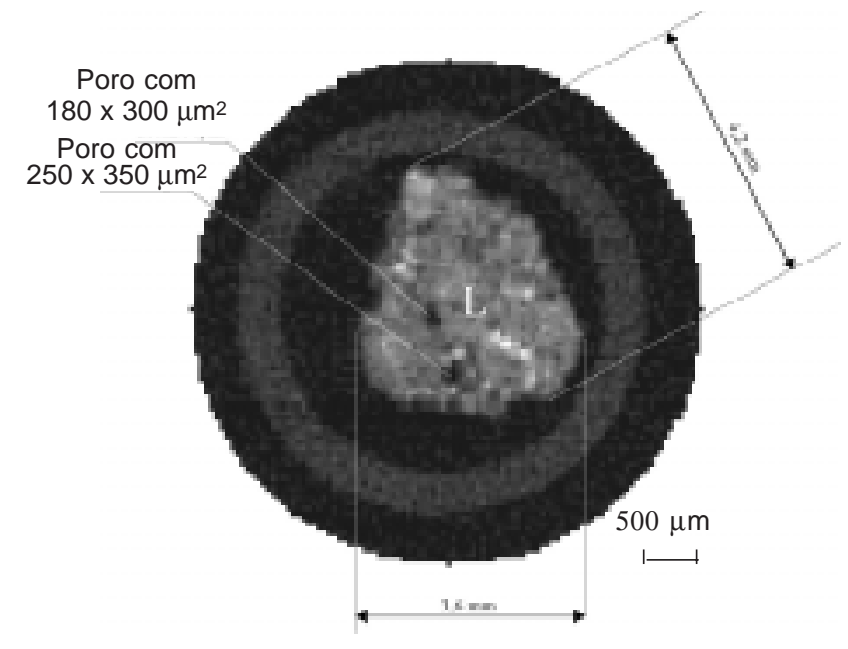

Figura 6. Tomografia de um torrão de Latossolo Vermelho-E scuro, onde podem ser vistos dois macroporos. $O$ valor máximo de densidade das partículas encontrado foi de $2,991 \mathrm{~g} \mathrm{~cm}^{-3}$.

(4) Amostra cedida pelo Prof. Dr. Orêncio Monje Vilar, do Departamento de Geotecnia, da Escola deEngenharia deSão Carlos, da Universidade de São Paulo. 


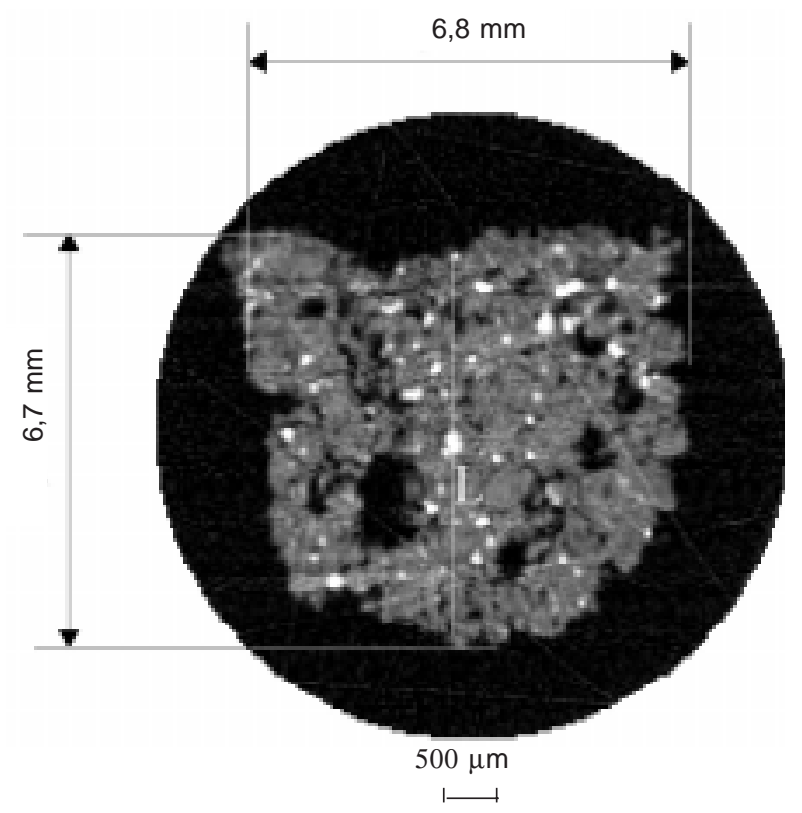

Figura 7. Microtomografia de um fragmento de torrão de solo, onde podem ser vistos poros de 200 a $800 \mu \mathrm{m}$ e pontos de alta atenuação, invisíveis em tomografia de resolução milimétrica. $O$ valor máximo de densidade das partículas encontrado foi de $4,005 \mathrm{~g} \mathrm{~cm}^{-3}$.

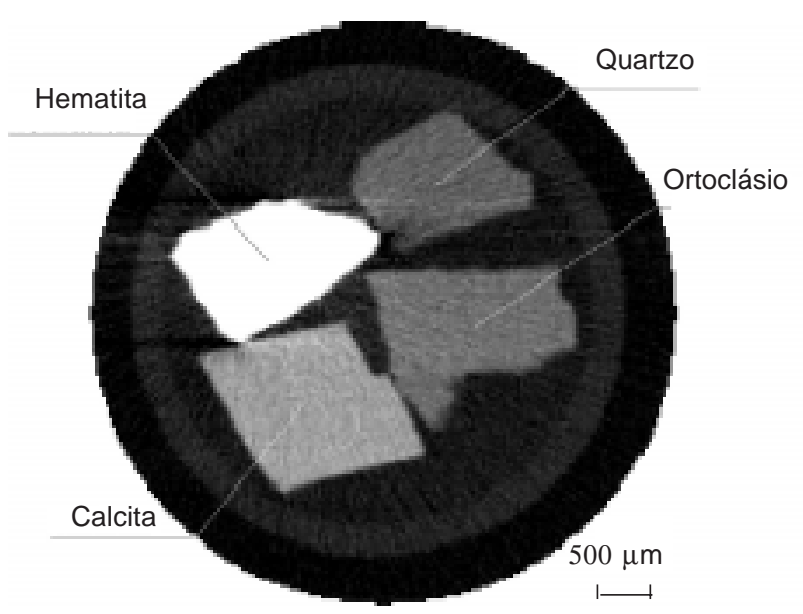

Figura 8. Tomografia de uma amostra composta por cristais de quartzo, ortoclásio, hematita e calcita, apresentando densidades reais, respectivamente, de 2,525, 2,586, 5,081 e $2,793 \mathrm{~g} \mathrm{~cm}^{-3}$. Os valores foram obtidos pelo método aqui proposto.

de escala (downscaling ou upscaling). Este desafio não é novo (Miller \& Miller, 1955a,b), mas se torna cada vez mais evidente, quando se começa a investigar o solo com técnicas e equi pamentos de imagem com alta resolução espacial, como no caso da
Quadro 2. Unidades tomográficas (UT), coeficiente de atenuação linear $(\mu)$ e comparação entre as densidades das partículas $\left(\rho_{s}\right)$ tabeladas e determinadas pelo método tomográfico

\begin{tabular}{lccccc}
\hline Cristal & UT & $\mu$ & $\rho_{\mathbf{s}}{ }^{(1)}$ & $\rho_{\mathbf{s}}{ }^{(2)}$ & Erro \\
\hline & & $\mathrm{cm}^{-1}$ & \multicolumn{2}{c}{$\mathrm{g} \mathrm{cm}^{-3}-$} & $\%$ \\
Quartzo & 0,682 & 0,665 & 2,650 & 2,525 & 4,7 \\
Ortoclásio & 0,784 & 0,764 & 2,570 & 2,586 & $-0,6$ \\
Hematita & 4,974 & 4,827 & 5,240 & 5,081 & 3,0 \\
Calcita & 1,132 & 1,101 & 2,730 & 2,793 & $-2,3$ \\
\hline
\end{tabular}

(1) Valores tabelados. ${ }^{(2)}$ Valores obtidos com o método aqui proposto.

microtomografia. Em outras palavras, o conceito de scaling precisa ser trazido à discussão, novamente, para se integrarem os resultados em várias escalas.

Como nova alternativa metodológica, a microtomografia pode associar-se a outras técnicas, estando entreelas, por exemplo, a porosimetria. Essa técnica enfrenta dificuldades de ordem prática na determinação do volume dos poros, tais como: o aprisionamento de bolhas de ar, a variação do volume, quando se utiliza água, e a fragilidade dos agregados do solo, quando se utiliza um líquido nãomolhante, como na porosimetria de Hg (Kutilek \& Nielsen, 1994).

Nas microtomografias com amostras de solos, foram visualizados pontos com alta densidade que não eram detectados na tomografia com resolução milimétrica. Na figura 7 , podem ser notados esses pontos, apresentando densidade de até $4,005 \mathrm{~g} \mathrm{~cm}^{-3}$. O mesmo não ocorre na tomografia milimétrica, mostrada na figura 9, onde se vê uma imagem da secção de uma amostra de sol o não perturbado. Essa imagem foi obtida em um tomógrafo portátil (Naime et al., 1996, 1997), com resolução milimétrica. Não há visualização dos referidos pontos nessa imagem, tampouco em outra do mesmo lote.

Outra característica que merece menção é a variação das unidades tomográficas que, na microtomografia, perde a característica de médias locais. A figura 10 mostra a variação das unidades tomográficas ao longo do transcepto $L$, da figura 7 , onde esse fato pode ser observado, quando em comparação com a figura 11, na qual se mostra a variação das unidades tomográficas da figura 9 . Na figura 11, nota-seapenas a curva oscilando em torno de um valor médiolocal, enquanto, na figura 10, temse uma informação muito mais detalhada sobre a amostra.

Cabemencionar queotomógrafo aqui apresentado tem a sua aplicação dirigida à caracterização de parâmetros ou fenômenos estáticos, pois os tempos deaquisiçãoestão na ordem de $50 \mathrm{~h}$. O desenvol vimento, 
tanto da técnica como do equi pamento, poderá trazer novos avanços e novas possibilidades. Está em andamento, no momento, a transformação do equipamento, passando de tomógrafo de primeira geração para tomógrafo de terceira geração, o que

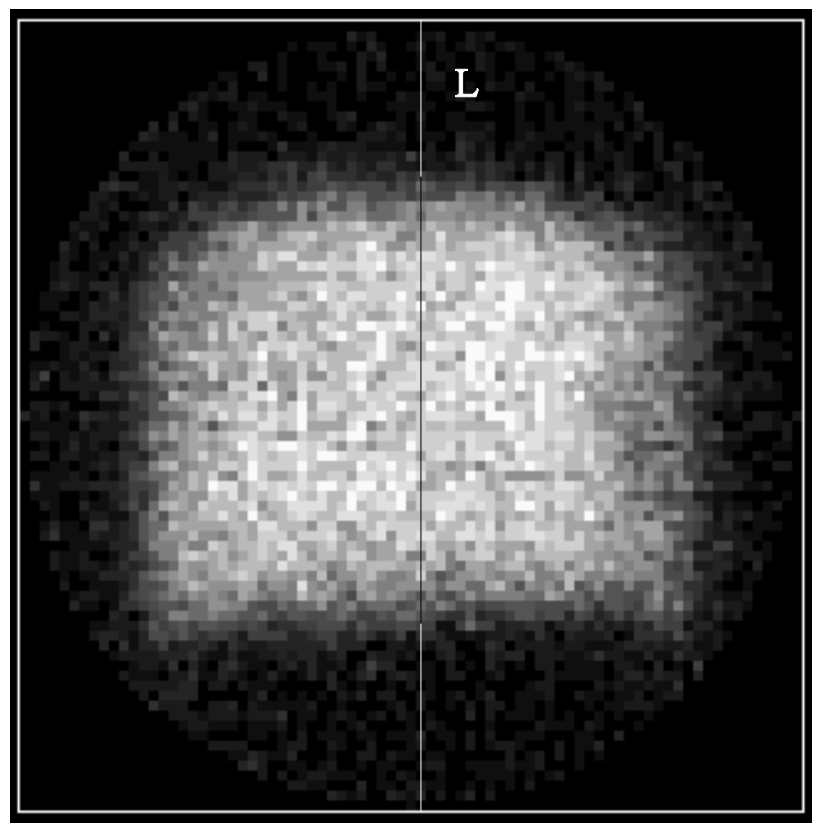

Figura 9. Tomografia em escala mi limétrica de uma amostra não perturbada de Podzólico VermelhoEscuro eutrófico, da Estação Experimental do IAC, em Mococa (SP).

Fonte: Dr. Carlos M.P. Vaz, da Embrapa Instrumentação Agropecuária.

TRANSCEPTO L

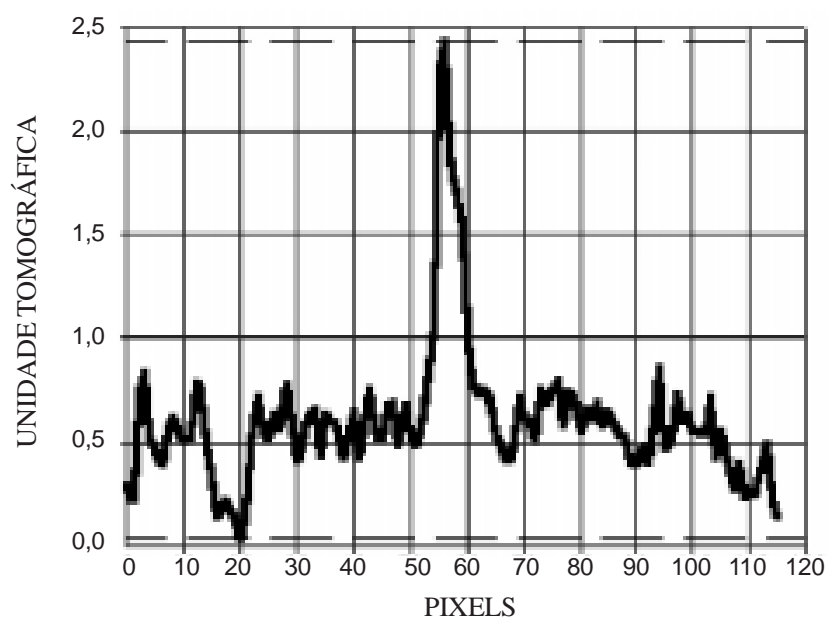

Figura 10. Variação das unidades tomográficas ao longo do transcepto $\mathbf{L}$ da microtomografia da figura 7.

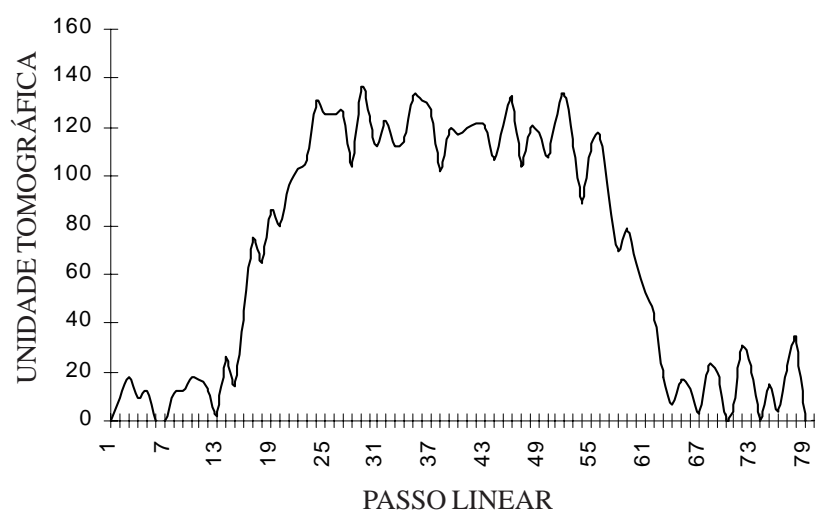

Figura 11. Variação das unidades tomográficas ao longo do transcepto $L$ da figura 9.

reduzirá esse tempo para em torno de $15 \mathrm{~min}$. I sto permitirá maior número de amostragens e, conseqüentemente, informações da variabilidade espacial da estrutura do solo. No estádio atual, a técnica já demonstra seu potencial de aplicações, apresentando resultados que antes não foram obtidos, pelo menos no que se refere à nãoinvasibilidade. Sua maior aplicação depende dos desenvolvimentos já citados e em andamento. Cabe citar, ainda, que entre eles encontra-se a implementação de ferramentas de análise de imagens, com o objetivo de avaliar a porosidade do solo, a partir das tomografias.

\section{CONCLUSÕES}

1. A construção do tomógrafo com resolução micrométrica, com aplicação em sol os, bem como em outros meios particulados, permite avaliar a densidade de partículas de sólidos com dimensões da ordem de $80 \mathrm{~mm}$ ou superior.

2. A microtomografia permiterealizar estudos de sistemas heterogêneos, porosos e opacos, como solos, com características inéditas na escala submilimétrica.

3. A visualização de macroporos e agregados do solo, trazendo informação mais detalhada sobre a amostra, coloca em discussãoa apl icação de conceitos da macroescala para mi oroescala, como, por exemplo, densidade e umidade do solo. Até o momento, não é óbvio que se possa fazer tal aplicação, sendo necessária, mais uma vez, a discussão do scaling.

\section{AGRADECIMENTOS}

À Embrapa/I nstrumentação Agropecuária (Projeto n-12.0.94.093) e à FAPESP (Processo 90/37737), pela infra-estrutura e pel o suporte financeiro. 
Ao Centro de Recursos Hídricos e Ecologia Aplicada da USP/Escola de Engenharia de São Carlos, onde foi desenvolvida a tese de doutorado.

Aos Assessores Científicos da Revista Brasileira de Ciência do Solo, pelos comentários que muito contribuíram para a melhoria deste trabalho.

\section{LITERATURA CITADA}

CESAREO, R.; CRESTANA, S. \& MASCARENHAS, S. Nuclear techniques in soil science. Trends in Agricultural Sciences: Soil Sci., 1:27-46, 1993.

CESAREO, R.; APPOLONI, C.R.; BRUNETTI, A.; CASTELLANO, A.; CRUVINEL, P.E.; MASCARENHAS, S.; ASSIS, J .T. \& GIGANTE, G.E. Industrial applications of tomography and microtomography. In: INTERNATIONAL SYMPOSIUM ON COMPUTERIZED TOMOGRAPHY FOR INDUSTRIAL APPLICATIONS, Berlin., 1994. Proceedings. Berlin, 1994. p.295-302.

CRESTANA, S.; MASCARENHAS, S. \& POZZI-MUCELLI, R.S. Static and dynamic three-dimensional studies of water in soil using computerized tomographic scanning. Soil Sci., 140:326-332, 1985.

CRESTANA, S.; CRUVINEL, P.E.; VAZ, C.M.P.; CESAREO, R.; MASCARENHAS, R. \& REICHARDT, K. Calibração e uso de um tomógrafo computadorizado em ciência do solo. R. Bras. Ci. Solo, 16:161-167, 1992.

CRESTANA, S. Técnicas recentes de determinação de características do solo. In: REUNIÃO BRASILEIRA DE MANEJ O E CONSERVAÇÃO DO SOLO E DA ÁGUA, 10., Florianópolis, 1994. Resumos. Florianópolis, Sociedade Brasileira de Ciência do Solo, 1994. p.86-97.

CRESTANA, S.; CRUVINEL, P.E.; MASCARENHAS, S.; BISCEGLI, C.I.; MARTIN-NETO, L. \& COLNAGO, L.A., eds. Instrumentação agropecuária: contribuições no limiar do novo século. Brasília, Empresa Brasileira de Pesquisa Agropecuária, 1996. 291p.

CRESTANA, S. \& NIELSEN, D.R. I nvestigações não-destrutivas de sistemas porosos multifásicos através de microtomografia de raios- $X$, gama e ressonância magnética nuclear (RMN). In: ENCONTRO NACIONAL SOBRE ESCOAMENTO EM MEIOS POROSOS-ENEMP, 18., Nova Friburgo, 1990. Anais. Nova Friburgo, Colégio Anchieta, 1990. p.699-710.

CRESTANA, S. \& VAZ, C.M.P. A instrumentação como uma ferramenta na pesquisa em conservação e manejo dos solos. In: CONGRESSO BRASILEIRO DE CIÊNCIA DO SOLO, 26., Rio de J aneiro, 1997. Resumos. Rio de J aneiro, 1997. CD-ROM.
CRUVINEL, P.E.; CESAREO, R.; CRESTANA, S. \& MASCARENHAS, S. X-ray and gamma-ray computerized minitomograph scanner for soil science. IEEE Trans. Instr. Meas., 39:745-750, 1990.

EMPRESA BRASILEIRA DE PESQUISA AGROPECUÁRIA EMBRAPA. Centro Nacional de Pesquisa de Solo. Manual de métodos de análise de solos. 2.ed. Rio de J aneiro, 1997. $212 p$.

FLANNERY, B.P.; DECKMAN, H.W.; ROBERGE, W.G. \& D'AMICO, K.L. Three-dimensional X-ray microtomography. Science, 237:1439-1444, 1987.

HAINSWORTH, J .M. \& AYLMORE, L.A.G. The use of computerassisted tomography to determine spatial distribution of soil water content. Aust. J . Soil Res., 21:1435, 1983.

KUTÍLEK, M. \& NIELSEN, D.R. Soil hidrology. CremlingenDestedt, Catena Verlag, 1994. 370p.

MACEDO, A.; CRUVINEL, P.E.; INAMASU, R.Y.; ORGE, L.A.C.; NAIME, J .M.; TORRE-NETO, A.; VAZ, C. M. P. \& CRESTANA, S. Micrometric X-ray CT Scanner dedicated to soil investigation. In: IEEE INTERNATIONAL MULTICONFERENCE ON CIRCUITS, SYSTEMS, COMMUNICATIONS AND COMPUTERS, 3., Atenas, 1999. (Trabalho completo aceito)

MILLER, E.E. \& MILLER, R.D. Theory of capillary flow: I. Experimental information. Soil Sci. Soc. Am. Proc. 19:271$275,1955 a$

MILLER, E.E. \& MILLER, R.D. Theory of capillary flow: I. Practical implications. Soil Sci. Soc. Am. Proc. 19:267-271, 1955b.

NAIME, J .M.; CRUVINEL, P.E. \& CRESTANA, S. Tomógrafo portátil dedicado à pesquisa agropecuária. São Carlos, EMBRAPA-CNPDIA, 1996. 4p. (EMBRAPA-CNPDIA, Comunicado Técnico, 11)

NAIME, J .M.; CRUVINEL, P.E.; CRESTANA, S.; CONCIANI, W. \& SOARES, M.M. Portable CAT scanner applied to collapsible soil studies. In: GAZZINELLI, R.; MOREIRA, R.L. \& RODRIGUES, W.N., eds. PHYSICS AND INDUSTRIAL DEVELOPMENT: BRIDGING THE GAP INTERNATIONAL CONFERENCE, 2., Belo Horizonte, 1997. Proceedings. Singapore, World Scientific, 1997. p.327331.

PETROVIC, A.M.; SIEBERT, J .E. \& RIEKE, P.E. Soil bulk density analysis in three dimensions by computed tomographic scanning. Soil Sci. Soc. Am. J ., 46:445-449, 1982.

ROSENFELD, A. \& KAK, A.C. Reconstruction. In: ROSENFELD, A. \& KAK, A.C. Digital picture processing. 2.ed. San Diego, Academic Press, 1982. v.1. p.353-430. (Computer Science and Applied Mathematics)

VAZ, C.M.P.; CRESTANA, S. \& REICHARDT, K. Tomografia computadorizada na avaliação da compactação de solos. R. Bras. Ci. Solo, 16:153-1159, 1992. 